\title{
Psychosocial impact on mothers receiving expanded newborn screening results
}

\author{
Kathleen O'Connor ${ }^{1} \cdot$ Tara Jukes $^{1} \cdot$ Sharan Goobie ${ }^{2}$ Jennifer DiRaimo ${ }^{2} \cdot$ Greg Moran $^{1} \cdot$ Beth Katherine Potter $^{3}$. \\ Pranesh Chakraborty ${ }^{4}$ Charles Anthony Rupar $^{2,5,6} \cdot$ Srinitya Gannavarapu ${ }^{2} \cdot$ Chitra Prasad $^{2,5}$
}

Received: 14 April 2017 / Revised: 20 October 2017 / Accepted: 18 November 2017 / Published online: 29 January 2018

(c) European Society of Human Genetics 2018

\begin{abstract}
Expanded newborn screening (NBS) for genetic disorders has improved diagnosis of numerous treatable diseases, positively impacting children's health outcomes. However, research about the psychological impact of expanded NBS on families, especially mothers, has been mixed. Our study examined associations between maternal experiences of expanded NBS and subsequent psychosocial functioning and parenting stress in mothers whose infants received either true negative (TN), true positive (TP) or false positive (FP) results after a 4- to 6-month period. The Parenting Stress Index and the Depression, Anxiety and Stress Scale were used to assess symptoms of anxiety, stress and depression in 3 sets of mothers, whose infants received TN $(n=31)$, TP $(n$ $=8)$ or FP $(n=18)$ results. Multivariate analyses of variance (MANOVA) results revealed no significant differences among these three groups of mothers regarding overall anxiety, stress and depression. However, FP mothers experienced lower levels of stress related to their own health compared to TN group. Two potential trends were also identified; results suggested TN mothers might experience higher levels of isolation than mothers in the TP group and that FP mothers might report higher stress levels in relation to spousal relationships compared to the TN group. FP mothers seemed to report similar or better levels of psychosocial functioning than TN mothers. Our findings are encouraging with respect to impacts of NBS on maternal well-being. We also identify key areas for improvement (parental education) and research (isolation and spousal relationships).
\end{abstract}

Kathleen O'Connor and Tara Jukes contributed equally to this work.

Electronic Database information URLs used in preparation of this article are Locus Link. National Center for Biotechnology Information, National Library of Medicine (Bethesda, MD), 1999. World Wide Web URL: https://www.ncbi.nlm.nih.gov/pubmed. Online Mendelian Inheritance in Man, OMIM (TM). Center for Medical Genetics, Johns Hopkins University (Baltimore, MD) and National Center for Biotechnology Information, National Library of Medicine (Bethesda, MD), 1999. World Wide Web URL: http://www.ncbi.nlm.nih.gov/ omim/.

Electronic supplementary material The online version of this article (https://doi.org/10.1038/s41431-017-0069-z) contains supplementary material, which is available to authorized users.

Publisher's note: Springer Nature remains neutral with regard to jurisdictional claims in published maps and institutional affiliations.

Chitra Prasad

chitra.prasad@lhsc.on.ca

1 Department of Psychology, Western University, London, ON, Canada

2 Department of Pediatrics, Western University, London, ON, Canada

3 School of Epidemiology, Public Health and Preventative

\section{Introduction}

Proposals for universal newborn screening (NBS) in Canada for genetic, metabolic and non-inherited disorders first emerged in the 1960s [1]. In the province of Ontario, the number of conditions routinely screened expanded from 2 to 29 between 2005 and 2013 through advances in screening technology and increased affordability [2-4]. Compared to children diagnosed after clinical presentation, infants identified through NBS are less likely to be hospitalized, experience shorter hospital stays, display $60 \%$ fewer medical problems and score 1-2 standard deviations higher on tests of infant development (motor, cognitive and language skills) and adaptive functioning $[3,5]$.

\section{Medicine, University of Ottawa, Ottawa, ON, Canada}

4 Newborn Screening, Children's Hospital of Eastern Ontario, Ottawa, ON, Canada

5 Children's Health Research Institute Ontario, London, ON, Canada

6 Department of Pathology and Laboratory Medicine, Western University, London, ON, Canada 
A small number of previous studies that focused on the psychosocial impact of NBS found that parents of children with conditions detected by NBS expressed lower stress and more satisfaction with their support systems and healthcare than those whose children were diagnosed with same conditions based on clinical symptoms (i.e., those whose children were not screened for the respective conditions) [3, 6]. There are clear medical and possibly parental psychosocial benefits of expanded NBS for children who have the screened diseases (i.e., those with true positive (TP) results). However, the significant number of false positive (FP) results generated by NBS are of concern in regards to the psychosocial impact these results may have on parents [7, 8]. The literature exploring the intensity and duration of impact of receiving FP screening results is mixed, with some studies suggesting that this experience may have an adverse effect on parents' well-being, including increased levels of guilt, poor self-esteem, anxiety and stigmatization $[9,10]$, and that FP results may impact impressions of one's child and the quality of the parent-child relationship [9, 11].

Specifically, some researchers have proposed that parents of children with FP results may be left with ongoing concerns about their child's health even though their child is not ultimately diagnosed with a genetic condition-perhaps because of misinformation about the results and their implications, or because they are not confident in their child's health moving forward [9, 11, 12]. Others have highlighted the possibility that FP results arising from expansions to the NBS panel may be linked with parental oversensitivity to physical and developmental symptoms and increased anxiety about parenting [13]. Though findings are varied, a recent study on NBS for medium-chain acyl-CoA dehydrogenase (MCAD) deficiency found increased physician and hospital visitations in FP infants [14]. Other studies have also found positive screening results (whether they are TP or FP after confirmatory testing) elicit clinically significant psychosocial impacts in the form of increased depressive symptoms and anxiety in the majority of parents $[15,16]$. Collectively, these findings, along with other research, suggest that positive NBS results that ultimately resolved as FP may have persisting negative psychosocial effects on families [11, 17, 18]. Additionally, the psychosocial well-being of parents of children with TP results has received little attention.

Given that a mother's thoughts, perceptions and beliefs regarding her child are considered foundational to the parent-child attachment relationship and subsequently shape child outcomes across numerous developmental domains [19], it is important to further efforts to clarify and evaluate mothers' experiences with the NBS process across all types of results. Our study thus examines the psychosocial well-being of mothers whose newborns received NBS and subsequently received either a FP, TP or true negative (TN) result. Mothers were divided into these three outcome groups and compared with regard to levels of depression, anxiety and both general and parenting-specific stress.

\section{Materials and methods}

\section{Sample and procedure}

This study was approved by the Health Sciences Ethics Review Board at Western University, London, Ontario, Canada. Informed consent was obtained from all participants. Mothers whose infants received an initial positive screen for an inborn error of metabolism, an endocrine disorder (congenital adrenal hyperplasia and congenital hypothyroidism) or cystic fibrosis through NBS were approached to participate in the study shortly after confirmatory testing was completed. This set of mothers was grouped based on the final diagnosis of their child into the TP or FP group. Mothers whose children screened negative were recruited through the Child Development Centre at Western University (London, Ontario, Canada) to form the TN group. These mothers were participating in an unrelated research project and had not participated in studies related to NBS. Mothers were initially contacted by either the treating physicians or the NBS coordinator at the London Health Sciences Center, London, Ontario, Canada. Some families chose not to participate in the study for a variety of reasons including time commitment and lack of interest. Only families that consented were followed after the initial contact. Consenting mothers were contacted by a member of the research team (who was not involved in the diagnosis and treatment) when the child was approximately 4-7 months of age. Our exclusion criteria included mothers with infants more than 8 months of age at the time of study and/or presenting with major congenital anomalies or serious health diagnosis unrelated to NBS.

Mothers in the positively screened groups (TP and FP) were offered the option of completing questionnaires at a designated university research space or receiving them through the mail to complete at home. Questionnaires administered at the university took approximately 45 to $60 \mathrm{~min}$ to complete. All mothers in the TN group completed their questionnaires at home, independently but in the presence of the questionnaire administrator. At the time of completing the questionnaires, all mothers were aware of their child's final diagnosis. No financial compensation was offered to mothers in the positively screened groups (TP and FP), but a small stipend was provided to the mothers in the TN group as part of a separate and unrelated study in which they participated. With no previous data available, we used a medium effect size $(f=0.25)$ as a conservative estimate to determine our sample size. We calculated 
that we would require a total sample size of 45 mothers, with $80 \%$ power and $5 \%$ significance.

\section{Measures}

The Depression, Anxiety and Stress Scale (DASS) [20] and the Parenting Stress Index (PSI) [21] are self-report inventories that were completed by all of the mothers. The correlation tables for each of these measures are provided (Supplementary Tables 13). The DASS consists of 3 subscales, each composed of 14 items, assessing depression, anxiety and stress, respectively. Each item is rated on a 4-point severity/frequency scale that describes the extent to which the state was experienced over the past week. Scores are calculated by summing scores of the relevant items within each subscale. The clinically normal ranges of scores, as determined by heterogenous population samples for the Depression, Anxiety and Stress subscales, are $0-9,0-7$ and $0-14$, respectively [20]. Higher scores (outside of the normal range) reflect greater clinical symptoms. The internal consistency (i.e., Cronbach's $\alpha$ ) for each subscale has been found to be high: DASS-Depression (0.96-0.97), DASSAnxiety (0.84-0.92) and DASS-Stress (0.90-0.95) [20].

The PSI measures stress related to parenting, relationships with spouse, family and friends, feelings of competence, social isolation and general health. It is composed of 101 Likert-scale items rated from 1 (Strongly Agree) to 5 (Strongly Disagree). This measure generates multiple subscale scores within two broad domains: Child Domain and Parent Domain. The Child Domain reflects stress related to the child's characteristics with the following subscales: Distractibility/Hyperactivity (assesses behavioral characteristics reflecting symptoms of attention deficit or hyperactivity disorder), Reinforces Parent (assesses parent's interactions with child as positively reinforcing), Mood (assesses child's affective status), Acceptability (assesses extent of child characteristics meeting parental expectations), Adaptability (assesses ability of child to change according to social and physical environments) and Demandingness (assesses parental experiences of child's demands). The Parent Domain captures stress related to the parenting role with the following subscales: Competence (assesses extent of parent's comfort and actual capability in parental role), Isolation (assesses degree of parent's social support), Attachment (assesses parent's sense of closeness with child), Health (assesses contribution of parent's heath to overall parenting stress), Role Restriction (assesses parent's sense of loss of identity and limited freedom), Depression (assesses parent's affective status) and Spouse (assesses parent's perception of support from spouse). Relevant items in each subscale are summed to generate scores for subscales. Total stress scores are derived by summing the Child Domain total score and Parent Domain total score. Total stress scores within the 16th-80th percentile (based on the consensus matched population of the United States) are considered to be clinically within a normal range [21]. Higher total scores and/or domain scores reflect greater stress related to that domain. The Total Stress scale and both domains (Parent and Child) have high internal consistency, with reliability coefficients of 0.96 or greater [21].

\section{Data analysis}

Outcomes of mothers in the TN, TP and FP groups were compared across three domains: (1) maternal social-emotional adjustment, specifically self-reported levels of depression, anxiety and stress (DASS); (2) perceptions of the child's functioning, level of distractibility, mood, acceptability, adaptability and demandingness (PSI-Child domain); (3) stress related to the parenting role, mothers' sense of competence as a parent, attachment, role restriction, depression, spousal support, isolation and health (PSI-Parent domain). All statistical analyses were done in SPSS (IBM Corp. Released 2012. IBM SPSS Statistics for Windows, Version 21.0, Armonk, NY). Preliminary analyses of all variables in the PSI and DASS had accepted distribution with only minor departures from normality in terms of skewness. Data were analyzed using multiple models of multivariate analyses of variance (MANOVAs) to allow group differences on all subscales within each of the three domains examined, without increasing risks of type I error. The $p$-values for the univariate analyses were corrected relative to the number of subscales in each domain (e.g., $p$ value for significance $=0.05$ /number of subscales). There was no case of large amounts of data missing from any mother; however, list-wise deletion was used when domains and scales contained incomplete information.

Based on previous studies which have identified links between maternal demographic factors (level of education, annual household income and marital status) and psychological functioning [22, 23], we examined these factors through preliminary correlational analyses to determine whether adjustment for these variables was required in the analysis to yield an unbiased estimate of the relationship between screening results and psychosocial outcomes. In our study, child age was variable within a small range and thus unlikely to act as a confounder, However, because there was a small age difference across the groups, we considered it as a potential covariate in our preliminary analyses to determine whether or not it might impact maternal stress, mood and adjustment to the parenting role.

\section{Results}

\section{Sample characteristics}

Of the 38 mothers approached for the positively screened group, 26 agreed to participate. These mothers were divided into two groups depending on the final confirmatory results 
their child received: TP $(n=8)$ and FP $(n=18)$. The babies of mothers in the TP group screened positive for and were ultimately diagnosed with the following conditions: cystic fibrosis, biotinidase deficiency, phenylketonuria, MCAD deficiency, argininosuccinate lyase deficiency and 3methylcrotonyl-CoA carboxylase deficiency. Mothers from the FP group had babies who screened positive for the following conditions/markers: elevated $\mathrm{C} 5 \mathrm{OH}, \mathrm{MCAD}$ deficiency, elevated C3 acylcarnitine (indicating an increased risk for propionic acidemia or methylmalonic acidemia), biotinidase deficiency, very long-chain acyl-CoA dehydrogenase deficiency, phenylketonuria, carnitine uptake deficiency, galactosemia, tyrosinemia and citrullinemia. The mean infant age at data collection was 6.87 months $(\mathrm{SD}=0.87)$. The highest education levels of the mothers include completion of high school or less education $(n=8)$, community college $(n=11)$, and a university or professional degree $(n=7)$. Eleven mothers (total $n=26$ ) reported an annual household income ranging from $\$ 50,000$ to $\$ 100,000 \mathrm{CDN}$. All but one reported English as the primary language spoken at home and all were fluent and able to complete the questionnaires. Two mothers reported having had another child who had also tested positive on newborn screening. Six mothers described themselves as healthcare professionals.

Responses from mothers of positively screened children were compared to the TN group of mothers $(n=31)$ whose infants screened negative through NBS. Mean infant age at the time of study participation was 4.57 months $(\mathrm{SD}=$ $0.44)$. Household incomes of mothers in the TN group ranged from $\$ 50,000$ to $70,000 \mathrm{CDN}$, with the majority having completed community college or some level of university. All mothers noted that English was the main language spoken at home. These mothers described their pregnancy and delivery as free from major complications and noted that their infants had been generally healthy since birth aside from minor ailments.

\section{Family demographic differences}

Correlation analyses indicated that there were no significant associations $(p>0.05)$ between each of the family demographic variables (education, socioeconomic status, income) and scores on the DASS and PSI. Even when associations with DASS and PSI domain scores were examined separately, no difference was identified. Thus, it was determined that family demographic variables would not need to be controlled for in subsequent analyses. Correlation analyses revealed no significant associations $(p>0.05)$ between infant's age, total scores on the DASS or PSI, or domain scores within these measures. Thus, child age was also not controlled for in the analyses described below. Rather, these variables were allowed to vary to maximize the generalizability and ecological validity of our results. The missing data percentages for the DASS, PSI-Child Domain and PSI-Parent Domain are $57.1 \%, 3.6-16.4 \%$ and $3.6-10.9 \%$, respectively. Descriptive statistics for all the variables assessed across questionnaires are presented in Table 1.

\section{Maternal social-emotional adjustment}

There was no significant variation at the multivariate level between groups on any domain of the DASS, Roy's largest $\operatorname{root}(0.08), \mathrm{F}(3,52)=1.45, p=0.238$. Due to DASS scores not varying across screening outcome groups (TN, TP, FP), univariate analyses were not explored. Descriptive statistics by screening outcome group are presented in Table 2 . Overall, the DASS scores from the TN, TP and FP groups did not indicate any clinically significant levels of depression, anxiety or stress (Table 2).

\section{Parenting stress-Child Domain}

A second MANOVA was conducted to examine variation across screening outcome groups on the Child Domain scales of the PSI. The multivariate analysis revealed significant variation across groups using Roy's largest root $(0.45), \mathrm{F}(6,45)=$

Table 1 Descriptive statistics reflecting parent ratings on study questionnaires across the TN, TP and FP groups

\begin{tabular}{|c|c|c|c|c|c|}
\hline & $N$ & Mean & SD & Minimum & Maximum \\
\hline \multicolumn{6}{|l|}{ DASS } \\
\hline Depression & 58 & 2.81 & 4.39 & 0 & 23 \\
\hline Anxiety & 56 & 3.02 & 4.78 & 0 & 24 \\
\hline Stress & 57 & 7.12 & 5.25 & 0 & 24 \\
\hline \multicolumn{6}{|l|}{ PSI-Child Domain } \\
\hline Distractibility & 53 & 22.96 & 2.84 & 17 & 28 \\
\hline Reinforcing & 57 & 8.28 & 2.88 & 6 & 22 \\
\hline Mood & 57 & 8.51 & 2.58 & 5 & 15 \\
\hline Acceptability & 56 & 10.13 & 2.58 & 7 & 16 \\
\hline Adaptability & 56 & 24.35 & 4.72 & 13 & 34 \\
\hline Demanding & 54 & 15.76 & 3.99 & 11 & 25 \\
\hline Child Total Stress & 52 & 90.31 & 14.85 & 64 & 125 \\
\hline \multicolumn{6}{|l|}{ PSI-Parent Domain } \\
\hline Competence & 53 & 25.43 & 4.97 & 14 & 38 \\
\hline Attachment & 56 & 11.88 & 3.52 & 7 & 21 \\
\hline Role restriction & 57 & 17.77 & 4.36 & 8 & 26 \\
\hline Depression & 55 & 17.40 & 4.55 & 9 & 29 \\
\hline Spousal support & 56 & 15.02 & 4.25 & 6 & 28 \\
\hline Isolation & 57 & 13.23 & 3.44 & 6 & 22 \\
\hline Health & 54 & 15.65 & 4.23 & 7 & 29 \\
\hline Parent total stress & 49 & 117.12 & 17.89 & 75 & 167 \\
\hline
\end{tabular}

The DASS and PSI scoring from all mothers is presented 
Table 2 Differences among TP, $\mathrm{FP}$ and TN groups on the DASS

\begin{tabular}{|c|c|c|c|c|}
\hline & \multicolumn{4}{|c|}{ Screening outcome groups } \\
\hline & $\begin{array}{l}\text { True negative }(N= \\
30) \text {, mean (SD) }\end{array}$ & $\begin{array}{l}\text { True positive }(N= \\
8), \text { mean }(\mathrm{SD})\end{array}$ & $\begin{array}{l}\text { False positive }(N= \\
18), \text { mean }(\mathrm{SD})\end{array}$ & Clinical significance \\
\hline \multicolumn{5}{|l|}{ DASS Score } \\
\hline Depression & $2.47(4.34)$ & $2.62(3.16)$ & $2.33(2.47)$ & Normal (TN, TP, FP) \\
\hline Anxiety & $3.40(4.92)$ & $1.38(1.30)$ & $3.11(5.52)$ & $\begin{array}{l}\text { Normal-mild (TN, FP) } \\
\text { normal (TP) }\end{array}$ \\
\hline Stress & $8.17(5.80)$ & $6.00(4.14)$ & $5.67(4.55)$ & Normal (TN, TP, FP) \\
\hline Total & $14.03(13.59)$ & $10.00(6.74)$ & $11.11(10.56)$ & Normal \\
\hline
\end{tabular}

Three variables were tested in the DASS. The above table displays the descriptive statistics for each group. DASS-Depression score ranges: normal (0-9), mild (10-13), moderate (14-20), severe (21-27) and extremely severe (28+) [20]. DASS-Anxiety: normal (0-7), mild (8-9), moderate (10-14), severe (15-19) and extremely severe (20+) [20]. DASS-Stress: normal (0-14), mild (15-18), moderate (19-25), severe (26-33) and extremely severe (34+) [20]
3.40, $p<0.01, \eta_{p}{ }^{2}=0.31$ (observed power $=0.91$ ). However, univariate analyses, at the corrected $p$-value (Table 3), revealed no significant differences $(p>0.008)$ between the groups in this domain. The Child Domain subscale scores were within the 16th-80th percentile, suggesting no clinically significant distress in the TN, TP and FP groups (Table 3).

\section{Parenting stress-Parent Domain}

A final MANOVA explored variation across screening outcome groups on the Parent Domain scales of the PSI. Roy's largest root $(0.87), \mathrm{F}(7,41)=5.09, p<0.01, \eta_{p}{ }^{2}=$ 0.47 (observed power $=0.99$ ) revealed significant variation across outcome groups. Univariate analyses, at the corrected $p$-value (Table 4) revealed significant differences between outcome groups on Health. The post hoc analyses using Tukey's honest significant difference (HSD) revealed that mothers of TN infants experienced significantly $(p<0.007)$ higher levels of stress related to their own health than those with FP infants or TP infants. Though not significant $(p>$ 0.007), two possible trends were identified between outcome groups on Spousal Support and Isolation. Mothers in the FP group seem to be more stressed regarding their relationships with their spouses compared to $\mathrm{TN}$ mothers. Additionally, TN mothers seem to experience higher levels of isolation than mothers in the TP group. The Parent Domain subscale scores were mostly within the 16th-80th percentile for the TN, TP and FP groups, suggesting no clinically significant distress, except for the Health subscale. The TN mothers reported within the 90th percentile, suggesting the mothers' concerns with their own health act as stressors impacting parenting behaviors (Table 4).

\section{Discussion}

Differences in psychosocial functioning of mothers of positively and negatively screened infants have not been studied extensively. Overall, our results indicated that mothers in the TP and FP groups reported similar or better psychosocial functioning 4 to 6 months after the NBS process compared to mothers in the TN group. Mothers in the TP and FP group reported similar levels of depression and general stress compared to the TN group. While these results appear to diverge from other research reporting that positive screens are linked with higher levels of psychological distress [17], it is important to note that several other studies provide support for the notion that parental distress related to FP results is short-lived [18, 24, 25]. For example, Kerruish [18] found that parents reported that their distress from receiving FP results was minimal and not long-lasting. It is also important to note that psychological distress and functioning are not the same. Psychological functioning can be described as an individual's ability to complete tasks set by themselves as well as individual behavior, emotional status, social skills and mental health. However, psychological distress is associated with the negative impact on an individual's level of functioning caused by unpleasant emotions.

\section{Impact of expanded NBS on parenting stress and the mother-child relationship}

Mothers in the FP group reported lower levels of parenting stress regarding their own health concerns compared to the TN group. Initially, our findings appear inconsistent with the study of Waisbren et al. [3] that found that parents of FP infants experienced higher overall levels of parenting stress and parent-child dysfunction 6 months following final diagnosis. However, that study's finding of greater stress was only true for families with a FP result who were not subsequently referred to a metabolic center. Genetics counselors in our NBS-related clinic at London Health Sciences Center offer counseling and education regarding positive screens during the initial appointment. Further, a management plan is implemented with the family while 
Table 3 Differences among TN, TP and FP groups on the Child Domain scales of the PSI

\begin{tabular}{|c|c|c|c|c|c|c|c|c|c|}
\hline \multirow{2}{*}{$\begin{array}{l}\text { PSI-Child } \\
\text { subscales }\end{array}$} & \multicolumn{9}{|c|}{ Screening outcome groups } \\
\hline & $\begin{array}{l}\text { True negative } \\
(N=26) \text {, mean } \\
(\mathrm{SD})\end{array}$ & TN-percentile & $\begin{array}{l}\text { True positive } \\
(N=8) \text {, mean } \\
(\mathrm{SD})\end{array}$ & TP-percentile & $\begin{array}{l}\text { False positive } \\
(N=18) \text {, mean } \\
(\mathrm{SD})\end{array}$ & FP-percentile & $\mathrm{F}(2,49)$ & $\begin{array}{l}\text { Sig. } \\
\text { ( } p \text {-value) }\end{array}$ & $\begin{array}{l}\text { Effect } \\
\text { size }\left(\eta_{p}{ }^{2}\right)\end{array}$ \\
\hline Distractibility & $22.85(2.99)$ & 45 & $23.12(3.04)$ & 45 & $23.39(2.33)$ & 45 & 0.20 & 0.817 & 0.01 \\
\hline Reinforcing & $7.96(1.99)$ & 45 & $9.13(5.89)$ & 55 & $8.67(2.45)$ & 55 & 0.58 & 0.565 & 0.02 \\
\hline Mood & $9.27(2.82)$ & 50 & $8.13(2.42)$ & 35 & $7.83(2.36)$ & 35 & 1.76 & 0.183 & 0.07 \\
\hline Acceptability & $10.23(2.63)$ & 30 & $9.88(2.64)$ & 30 & $9.94(2.75)$ & 30 & 0.09 & 0.916 & 0.00 \\
\hline Adaptability & $25.85(4.27)$ & 65 & $24.25(3.69)$ & 50 & $22.11(5.35)$ & 35 & 3.51 & 0.038 & 0.13 \\
\hline Demanding & $16.42(3.85)$ & 35 & $15.50(5.24)$ & 35 & $15.22(3.81)$ & 25 & 0.50 & 0.608 & 0.02 \\
\hline
\end{tabular}

Six variables were tested in the PSI Child Domain for significance at $p<0.008$ (corrected for number of dependent variables (i.e., subscales), please refer to text for details). No significant differences were found between the three groups of mothers (TN, TP, FP). PSI-Child score percentile ranges: normal (16th-80th), borderline (81st-84th) and clinically significant (85th-99+) [21]. Note that the subscales labeled Acceptability and Adaptability are reverse coded, meaning a higher score reflects lower acceptability and adaptability

they wait for confirmatory results. The family is connected with at least one member of the healthcare team and have access to them at any time of any day should they have concerns. This aspect of our study is crucial as some earlier work has suggested that characteristics of the healthcare system within which NBS is executed may have an important impact on how this process is perceived by parents. Studies indicate that high-quality parental education and communication may have a positive impact on parents' stress and anxiety [13], including those who receive FP results [17]. Our findings suggest that the execution of our NBS program likely impacts the experience perceived by the mothers. The study of Waisbren et al. [3] also used a short form of the PSI, which may not have captured as comprehensive a picture of parental adjustment as measures used in our study.

Several previous studies have suggested that FP results may lead to heightened concern and biased parental perception about the children's health and well-being over time $[3,9,10,13,18]$. Our findings were not supportive of this notion. Overall, mothers of FP children did not report increased challenges, difficulties or concerns about their children relative to mothers in the TN or TP groups. Our results suggest a potential trend in TP and FP mothers describing their children as more adaptable than mothers in the TN group. These findings are consistent with at least one other qualitative study that found that relief experienced by parents following confirmation that their child's result was FP contributed to a new perspective on parenting that included appreciation of their child's good health $[8,25]$. It may be that ongoing stress associated with FP results found in previous studies is very specific to future health concerns which may not have been captured in our study. However, our results suggest that these concerns do not appear to extend to perceptions of overall child behavior and parent psychosocial functioning.

\section{Impact of FP results on spousal relationships}

Our results suggest a possibility that FP results might negatively impact spousal relationships. Studies from other areas of children's health may shed light on this result. For example, research has suggested that parents coping with a childhood cancer diagnosis experience relationship challenges associated with stress and uncertainty in their child's prognosis [26]. Contrastingly, Tluczek et al. [8] reported that while parents whose infants had screened FP for cystic fibrosis experienced some negative effects (e.g., frustration with the process, guilt, concern about future reproductive decisions), they also felt that the experience had brought them closer to their spouse, strengthened ties with extended family members and increased levels of gratitude and appreciation for their child's well-being. Alternatively, there is some evidence from the NBS literature that mothers and fathers cope differently with the screening process [8]. Given our study's smaller sample size, we were unable to determine a significant difference between outcome groups for spousal support and we acknowledge that this difference may have arisen due to chance. However, it is possible that the trend we noticed may be a result of differences in coping ability in mothers awaiting the final diagnosis of their child, contributing negatively to their marital relationship.

\section{Longitudinal impact of NBS}

Our findings largely support the notion that there are few significant long-standing psychosocial challenges for mothers associated with receiving TP or FP results [25, 27]. Through the course of our study, we observed a potential need for establishing prenatal and ongoing support and counseling for families. It is interesting to note that a concern of our physicians and genetic counselors has been the lack of education about NBS and its objectives during 
Table 4 Differences among TN, TP and FP groups on the Parent Domain scales of the PSI

\begin{tabular}{|c|c|c|c|c|c|c|c|c|c|}
\hline \multirow{2}{*}{$\begin{array}{l}\text { PSI-Parent } \\
\text { subscales }\end{array}$} & \multicolumn{9}{|c|}{ Screening outcome groups } \\
\hline & $\begin{array}{l}\text { True negative } \\
(N=23) \text {, mean } \\
(\mathrm{SD})\end{array}$ & TN-percentile & $\begin{array}{l}\text { True positive } \\
(N=8) \text {, mean } \\
(\mathrm{SD})\end{array}$ & TP-percentile & $\begin{array}{l}\text { False positive } \\
(N=18) \text {, mean } \\
(\mathrm{SD})\end{array}$ & FP-percentile & $\mathrm{F}(2,46)$ & $\begin{array}{l}\text { Sig. } \\
\text { ( } p \text {-value })\end{array}$ & $\begin{array}{l}\text { Effect } \\
\text { size }\left(\eta_{p}{ }^{2}\right)\end{array}$ \\
\hline Competence & $26.52(5.20)$ & 40 & $25.00(6.00)$ & 30 & $24.72(4.17)$ & 30 & 0.73 & 0.487 & 0.03 \\
\hline Attachment & $12.30(3.40)$ & 50 & $13.50(5.76)$ & 75 & $11.33(2.38)$ & 35 & 1.07 & 0.351 & 0.04 \\
\hline Role restriction & $18.43(4.87)$ & 45 & $16.63(4.53)$ & 40 & $18.11(3.81)$ & 45 & 0.50 & 0.612 & 0.02 \\
\hline Depression & $18.17(5.33)$ & 35 & $15.63(5.04)$ & 20 & $17.56(3.65)$ & 35 & 0.86 & 0.429 & 0.04 \\
\hline Spousal support & $13.17(4.06)$ & 25 & $15.38(3.93)$ & 40 & $17.33(4.01)$ & 55 & 5.42 & 0.008 & 0.19 \\
\hline Isolation & $14.39(3.27)$ & 70 & $10.88(3.56)$ & 35 & $13.06(3.44)$ & 60 & 3.31 & 0.046 & 0.13 \\
\hline Health & $17.57(4.21)$ & 90 & $12.38(3.85)$ & 65 & $14.06(3.08)$ & 75 & $7.50 *$ & 0.002 & 0.25 \\
\hline
\end{tabular}

Among the 7 tested variables, Health was found to be significantly different between different result groups. TN mothers reported higher concerns regarding their own health compared to TP mothers. *Statistical significance at $p<0.007$ (corrected for number of dependent variables (i.e., subscales), please refer to text for details). PSI-Parent score percentile ranges: normal (16th-80th), borderline (81st-84th) and clinically significant $(85$ th-99+) [21]

prenatal care. The observations of our participating physicians suggest increased prenatal education for all expectant mothers might mitigate some of the initial apprehension and confusion towards NBS. Though this aspect was not explored in detail in this study, further research examining the educational impact on psychosocial functioning of parents of positively screened newborns is warranted.

\section{Study limitations}

Primarily, all participants were identified and followed-up through a local children's hospital and these findings can only be generalized to families seen in similar settings [3]. Additionally, only mothers were included in our study for logistical reasons. Although the cascading impact of a psychologically affected mother on other family members is accepted [28], understanding the distinct paternal aspect of NBS is still important but has not been captured by our measures. Examining the associations between NBS results and paternal psychosocial and marital functioning is an important direction for future research. It is also important to note that though our sample size of mothers satisfied the requirements of our power analysis, a larger sample might have shed more light on the two trends we noticed in the Parent Domain of the PSI. Another point of importance is that we did have a small percentage (still within an acceptable range) of missing data as mentioned earlier that needs to be considered when interpreting the results in this study.

\section{Conclusion}

Expansion of NBS programs has improved identification and treatment of several genetic and metabolic disorders via early intervention. Our study findings provide an encouraging and positive evaluation of the psychosocial impact of the current NBS program. They play an important role in highlighting that NBS as it currently exists in Ontario may not have as detrimental an impact on maternal psychosocial well-being and functioning as originally envisioned by some. We also identify a few key areas for further research and intervention: the marital relationship, paternal NBS experiences and continual parental education. Understanding the experiences of both parents is important in assessing the impact of FP and TP results on martial relationships and resulting changes in family dynamics. Additionally, understanding the impact of ongoing support and counseling, as reported by FP mothers, is integral to redesign current NBS practices to incorporate additional resources and support for parents of positively screened newborns. Our work provides further support for the positive implications of expanded NBS and emphasizes the importance of a more comprehensive, balanced system that supports the physical, emotional and relational aspects of individual and family functioning.

Acknowledgements We thank all the families for their participation. We thank Dr. Michael Miller for his guidance in conducting the statistical analyses and thoughtful discussions.

Funding This research was funded by Newborn Screening Ontario, Children's Hospital of Eastern Ontario, Ottawa, ON, Canada.

\section{Compliance with ethical standards}

Conflict of interest The authors declare that they have no conflict of interest.

\section{References}

1. Hanley WB. Newborn screening in Canada - are we out of step? Paediatr Child Health (Oxf). 2005;10:203-7. 
2. Dhondt J-L. Expanded newborn screening: social and ethical issues. J Inherit Metab Dis. 2010;33:S211-S217.

3. Waisbren SE, Albers S, Amato S, et al. Effect of expanded newborn screening for biochemical genetic disorders on child outcomes and parental stress. JAMA. 2003;290:2564-72.

4. BORN Ontario. Born \& Growing Annual Report 2012-2014. 2014: 1-56.

5. Potter BK, Chakraborty P, Kronick JB, et al. Achieving the 'triple aim' for inborn errors of metabolism: a review of challenges to outcomes research and presentation of a new practice-based evidence framework. Genet Med. 2013;15:415-22.

6. Waisbren SE, Weipert CM, Walsh RC, Petty CR, Green RC. Psychosocial factors influencing parental interest in genomic sequencing of newborns. Pediatrics. 2016;137:S30-S35.

7. Gurian EA. Expanded newborn screening for biochemical disorders: the effect of a false-positive result. Pediatrics. 2006;117:1915-21.

8. Tluczek A, Orland KM, Cavanagh L. Psychosocial consequences of false-positive newborn screens for cystic fibrosis. Qual Heal Res. 2011;21:174-86.

9. Kerruish NJ. Parents' experiences of newborn screening for genetic susceptibility to type 1 diabetes. J Med Ethics J Inst Med Ethics. 2011;37:348-53.

10. Tarini BA. Communicating with parents about newborn screening: the skill of eliciting unspoken emotions. Arch Pediatr. 2012;166:95-96.

11. Rueegg CS, Barben J, Hafen GM, et al. Newborn screening for cystic fibrosis - the parent perspective. J Cyst Fibros. 2015;15:443-51.

12. Ulph F, Cullinan T, Qureshi N, Kai J. Parents' responses to receiving sickle cell or cystic fibrosis carrier results for their child following newborn screening. Eur J Hum Genet. 2015;23:459-65.

13. Bailey DB, Armstrong FD, Kemper AR, Skinner D, Warren SF. Supporting family adaptation to presymptomatic and 'untreatable' conditions in an era of expanded newborn screening. J Pediatr Psychol. 2009;34:648-61.

14. Karaceper MD, Chakraborty P, Coyle D, et al. The health system impact of false positive newborn screening results for mediumchain acyl-CoA dehydrogenase deficiency: a cohort study. Orphanet J Rare Dis. 2016;11:12

15. Beucher J, Leray E, Deneuville E, et al. Psychological effects of false-positive results in cystic fibrosis newborn screening: a twoyear follow-up. J Pediatr. 2010;156:771-6.
16. Tluczek A. Psychosocial risk associated with newborn screening for cystic fibrosis: parents' experience while awaiting the sweattest appointment. Pediatrics. 2005;115:1692-703.

17. Hewlett J, Waisbren SE. A review of the psychosocial effects of false-positive results on parents and current communication practices in newborn screening. J Inherit Metab Dis. 2006;29:677-82.

18. Kerruish N. Parents' experiences 12 years after newborn screening for genetic susceptibility to type 1 diabetes and their attitudes to whole-genome sequencing in newborns. Genet Med. 2015;18:249-58.

19. Sroufe LA. Attachment and development: a prospective, longitudinal study from birth to adulthood. Attach Hum Dev. 2005;7:349-67.

20. Lovibond SH, Lovibond PF. Manual for the Depression Anxiety Stree Scales. 2nd ed. Sydney, NSW, Australia: Pyschology Foundation; 1995.

21. Abidin RR. Parenting Stress Index. 3rd ed. Odessa, FL: Pyschological Assessment Resources; 1995.

22. Jackson B, Goodman E. Low social status markers: do they predict depressive symptoms in adolescence? Race Soc Probl. 2011;3:119-28.

23. Elovainio M, Pulkki-Råback L, Jokela M, et al. Socioeconomic status and the development of depressive symptoms from childhood to adulthood: a longitudinal analysis across 27 years of follow-up in the Young Finns study. Soc Sci Med. 2012;74:923-9.

24. Rowe H, Fisher J, Quinlivan J. Women who are well informed about prenatal genetic screening delay emotional attachment to their fetus. J Psychosom Obstet Gynecol. 2009;30:34-41.

25. Schmidt JL, Castellanos-Brown K, Childress S, et al. The impact of false-positive newborn screening results on families: a qualitative study. Genet Med. 2012;14:76-80.

26. Da Silva FM, Jacob E, Nascimento LC. Impact of childhood cancer on parents' relationships: an integrative review. J Nurs Scholarsh. 2010;42:250-61.

27. Tu WJ, He J, Chen H, Shi XD, Li Y. Psychological effects of false-positive results in expanded newborn screening in China. PLoS ONE. 2012;7:e36235.

28. Frankel LA, Pereira S, McGuire AL. Potential psychosocial risks of sequencing newborns. Pediatrics. 2016;137:S24-29. 